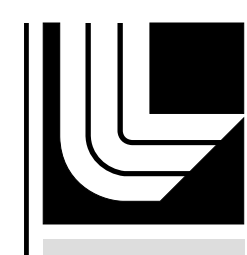

LAWRENCE LIVERMORE N A T IO N A L LABORATORY

Line-imaging velocimeter for shock diagnostics at the OMEGA laser facility

P. M. Celliers, D. K. Bradley, G. W. Collins, D. G. Hicks, T. R. Boehly, W. J. Armstrong

February 5, 2004

Review of Scientific Instruments 
This document was prepared as an account of work sponsored by an agency of the United States Government. Neither the United States Government nor the University of California nor any of their employees, makes any warranty, express or implied, or assumes any legal liability or responsibility for the accuracy, completeness, or usefulness of any information, apparatus, product, or process disclosed, or represents that its use would not infringe privately owned rights. Reference herein to any specific commercial product, process, or service by trade name, trademark, manufacturer, or otherwise, does not necessarily constitute or imply its endorsement, recommendation, or favoring by the United States Government or the University of California. The views and opinions of authors expressed herein do not necessarily state or reflect those of the United States Government or the University of California, and shall not be used for advertising or product endorsement purposes. 
pmc-omega-visar-v3

\title{
Line-imaging velocimeter for shock diagnostics at the OMEGA laser facility
}

\author{
P.M. Celliers, D.K. Bradley, G.W. Collins, and D.G. Hicks \\ Lawrence Livermore National Laboratory, Livermore, CA 94550 \\ T.R. Boehly and W.J. Armstrong \\ Laboratory for Laser Energetics, University of Rochester, Rochester, NY
}

(Dated: January 15, 2004)

\begin{abstract}
A line-imaging velocity interferometer has been implemented at the OMEGA laser facility of the Laboratory for Laser Energetics, University of Rochester. This instrument is the primary diagnostic for a variety of experiments involving laser-driven shock wave propagation, including high pressure equation of state experiments, materials characterization experiments, shock characterization for Rayleigh-Taylor experiments, and shock timing experiments for inertial confinement fusion research. Using a laser probe beam to illuminate a target the instrument measures shock breakout times and Doppler shifts in the reflected light. Velocities of interfaces, free surfaces and of shock fronts traveling through transparent media can be measured for velocities ranging from 0.1 to greater than $50 \mathrm{~km} / \mathrm{s}$ with accuracies $\sim 1 \%$ over most of this range. Quantitative measurements of the optical reflectance of ionizing shock fronts can also be obtained simultaneous with the velocity measurements.
\end{abstract}




\section{INTRODUCTION}

Optical diagnostics are the principal means for observing shock-wave experiments generated on large laser facilities. The earliest laser-driven shock wave experiments studied shock propagation by detecting the thermal luminescence produced when a strong shock breaks through the surface of an opaque stepped target $[1,2]$. This technique requires the shock to be sufficiently strong that the thermal luminescence is readily detectable after breakout of the shock front; it has been utilized in a number of shock detection studies at high energy laser facilities[3-7]. Dedicated instruments to perform this kind of measurement have been developed at the NOVA[8] and OMEGA[9] laser facilities.

Active shock detection techniques probe the target with a laser beam and observe, for example, a sharp change in the optical reflectance when a strong shock breaks through the rear surface[10, 11]. Since signal levels are determined by the probe intensity, not by the target luminescence, active probing techniques can be made sensitive to shock strengths that are too weak to produce a strong luminescence signal (usually less than $100 \mathrm{GPa}$ ), but also to ultra high pressure shocks in the TPa range. Active probing techniques can provide a wealth of information on transport properties, primarily through quantitative measurement of optical reflectance. Measurements of target reflectance with active probes have revealed detailed information on electrical conductivities in release profiles[12] and in the shock front $[13,14]$.

An obvious development path for active probing methods is to combine them with interferometric detection schemes. Interferometric detection schemes have long been available for shock wave studies: displacement and velocity interferometry techniques were first developed on gas gun and explosively-driven shock facilities by Barker and others in the 1960s and 1970s[15-19] leading to the establishment of the velocity interferometer system for any reflector (VISAR)[17] as a standard high-precision velocity diagnostic. The closely related optically-recording velocity interferometer system (ORVIS)[20] employed a streak camera to record the data with sub-nanosecond time resolution. Later, line-imaging variations on these methods [21-25] took advantage of the spatially resolving capabilities of streak camera detectors to encode spatial information. Adapting these techniques to the context of laserdriven targets $[23,24,26,27]$ has enabled precise measurement of the motion of a variety of shock-related phenomena in laser-driven targets, including the motion of free surfaces, of 
shocked interfaces, and of ionizing shock fronts in a wide variety of transparent media[28-30].

The active shock breakout system (ASBO) system at the OMEGA laser of the Laboratory for Laser Energetics (LLE) in Rochester, NY is a line-imaging velocity interferometer that is used as a general purpose shock diagnostic. In opaque targets it is used to measure shock speeds by detecting breakout times across stepped interfaces at high shock pressures $(\geq$ $100 \mathrm{GPa}$ ), or by detecting the motion of solid and optically reflecting free surfaces at lower

shock pressures (few 10s of GPa). In transparent targets it can measure the velocity of reflecting shock fronts directly from the Doppler shift (usually $>100 \mathrm{GPa}$ ), or the velocity of an interface behind a transparent window material at lower stresses (e.g. up to $200 \mathrm{GPa}$ behind a LiF window). The instrument can also be used to determine the target reflectance for many experimental situations. The development of this instrument began on the NOVA laser facility at Lawrence Livermore National Laboratory[24]. The design was improved and transferred to the OMEGA laser facility at LLE. Most elements of this design have proven versatile and portable - variations on it have been implemented at the Phebus[31], Vulcan[32] and LULI[33] facilities. In this article we describe in detail the design, implementation and use of this diagnostic at the OMEGA laser facility. We present examples of data collected during recent experiments on the OMEGA laser.

\section{SYSTEM LAYOUT}

\section{A. Overview}

At its simplest level the ASBO diagnostic is a high resolution optical imager that projects a two-dimensional magnified image of the target onto a pair of streak camera detectors (Fig. 1). In somewhat more detail, the system can be divided into a cascade of image relays leading from the target chamber through a ten-inch diagnostic manipulator (TIM) and then into the detection paths. The signal passes through a pair of velocity interferometers before being recorded on the streak cameras. The interferometers superimpose a sinusoidal spatial modulation on the image: Doppler shifts in the reflected probe are manifested as shifts of these fringes at the interferometer outputs. The streak cameras record the central chord of the field of view and sweep this signal in time across the output detector.

The probe light is delivered to the diagnostic system through a large core diameter 
multimode fused silica optical fiber, the output of which is collimated and injected into the optical system through a $50 \%$ beam splitter. Light reflected from the target returns to this beam splitter from which it is reflected into the detection paths.

\section{B. Image Relays}

\section{Target to interferometer}

The first part of the imaging system, shown in Fig. 2a, is a pair of telescopes cascaded to produce a magnified real image at the interferometers. The optical elements of this relay comprise a $146 \mathrm{~mm}$ objective (L1/L2), a $1675 \mathrm{~mm}$ plano-convex singlet (L3, CVI PLCX-1675-50.8), a 1000 mm achromat (L4, CVI AAP-1000-50.8), and a 1675 mm planoconvex (L5, identical to L3). The objective is a compound system with two elements: a $350 \mathrm{~mm}$ meniscus (L1, Melles Griot LMP 031) and a $250 \mathrm{~mm}$ achromat (L2, CVI AAP250-50.8). This combination has $146 \mathrm{~mm}$ focal length and focal ratio $\mathrm{F} / 3.3$. The meniscus also acts as a blast shield to protect the more expensive achromat from target debris, and is replaced every shot. While not achromatic the L1/L2 combination has close to diffraction limited performance for the monochromatic probe laser. The point spread resolution at $532 \mathrm{~nm}$ is around $4 \mu \mathrm{m}$. The objective collimates the image of the target and transmits it approximately $1 \mathrm{~m}$, where it passes through lens L3. The elements, L1, L2 and L3 are rigidly mounted on the ends of a tube and inserted into the target chamber as a unit. Focusing is achieved by slight axial translations of this tube. An intermediate image plane is situated beyond L3 inside the vacuum housing approximately $1 \mathrm{~m}$ from the vacuum window. The next element in the relay is the $1000 \mathrm{~mm}$ achromat L4, which also functions as the vacuum window. L4 re-collimates the beam for the path segment leading from the vacuum housing and through a periscope system onto the diagnostic table (Fig. 2b) where it reaches L5. A set of two beam splitters and a mirror are placed in the paths beyond lens L5. The elements L1 - L5 are $50 \mathrm{~mm}$ diameter.

The first beam splitter B1 is required to allow injection of the probe beam into the optical system, and is approximately 50\% reflecting system at $532 \mathrm{~nm}$. The output images are formed $1500 \mathrm{~mm}$ beyond lens L5 and the interferometers are placed precisely such that these images are simultaneously coincident with the output beam splitter in each interferometer. 
Magnification at the intermediate image plane inside the vacuum housing is $\sim 10 \mathrm{x}$ (given by the ratio of focal lengths of L3 and L1/L2), while magnification in the second stage of the relay from intermediate image plane to the interferometer plane is $\sim 1.5 \mathrm{x}$, yielding a total magnification from target to interferometer of $15.3 \mathrm{x}$.

\section{Interferometer to streak camera}

The interferometers are adjusted to impose a fringe pattern on the the beam passing through them, and are aligned such that the fringes are localized at the output-beamsplitter plane, coincident with the image formed by L5. The final relay, comprising $300 \mathrm{~mm}$ achromats L6A and L6B (CVI AAP-300.0-50.8) and $500 \mathrm{~mm}$ achromats L7A and L7B (CVI AAP-500.0-50.8), is laid out in a folded arrangement with the $300 \mathrm{~mm}$ achromat placed one focal length from the output beam splitter to collimate the image, and the $500 \mathrm{~mm}$ element placed one focal length from the streak camera slit to reform the image. The fold mirrors (M2 - M7) in these final relays are $76 \mathrm{~mm}$ diameter.

A pair of narrow band $(3 \mathrm{~nm})$ interference filters are placed in this leg to block thermal emission and any residual $527 \mathrm{~nm}$ emission originating from the OMEGA laser. Additional optional neutral density attenuating filters are also placed here to balance signal levels on the streak cameras.

Total magnification in this stage is $1.67 \mathrm{x}$, giving a system magnification from the target to the streak camera slit of $25.5 x$. The effective field of view at the target plane is approximately $760 \mu \mathrm{m}$. At 25.5x magnification the $4 \mu \mathrm{m}$ point spread function at the object plane is well matched to the point spread function of the streak tube $(\sim 100 \mu \mathrm{m})$, and results in $\sim 200$ spatial resolution elements across the field of view.

\section{Interferometers}

The interferometers employ a Mach-Zehnder configuration, shown in Fig. 3, and are constructed on $19 \mathrm{~mm}$ thick aluminum plates placed on three-point kinematic supports. Several interferometer plates are configured at any given time, and the kinematic mounting allows the interferometers to be changed with relative ease between experiments, thus allowing variability in the velocity sensitivities. The distance between the end mirrors is approximately 
$500 \mathrm{~mm}$, the lateral separation of the beam splitters is approximately $40 \mathrm{~mm}$, and the angle enclosed between the folded beams is approximately 9 degrees. The optical elements in the interferometers are $25.4 \mathrm{~mm}$ diameter: a pair of protected silver mirrors and a pair of $50 \%$ beam splitters optimized for $532 \mathrm{~nm}$ operation. The interferometer is designed to produce a precise delay in one of its arms. The end mirror and the etalon in the delay arm of the interferometer are both mounted on a motorized translation stage. The encoded motor can move the stage to arbitrary positions with $\mu \mathrm{m}$ precision. The optical delay is achieved by a combination of the refractive delay in the etalon and an additional translational offset of the etalon-mirror combination along a direction perpendicular to the mirror plane. The translation distance, $d=h(1-1 / n)$, is calculated to place the image of the mirror plane as viewed through the etalon coincident with its initially-determined null position (i.e. zero path delay with the etalon removed, see below and appendix). The resulting optical time delay is[17, 34],

$$
\tau=\frac{2 h}{c}(n-1 / n),
$$

where $h$ is the etalon thickness, $n$ is the index of refraction of the etalon and $c$ is the speed of light. The etalon elements are made of UV-grade fused silica with anti-reflection coatings for $532 \mathrm{~nm}$ on both surfaces. In some cases a delay of a given thickness is achieved by stacking two or more such pieces into the mounting barrel[35]. Total etalon thickness can vary from $2 \mathrm{~mm}$ up to $30 \mathrm{~mm}$, corresponding to velocity sensitivities ranging from $25 \mathrm{~km}$ $\mathrm{s}^{-1}$ fringe ${ }^{-1}$ to $1.7 \mathrm{~km} \mathrm{~s}^{-1}$ fringe ${ }^{-1}$, respectively (see section IV below).

\section{Setup and alignment}

Alignment and installation of etalons in the interferometers is accomplished at a workstation away from the target area. The alignment procedure is designed to produce a setting for the optical delay that is accurate to a few microns in optical path; this is critical for ensuring the accuracy of the measurements. The aligment procedure also ensures that the optical axis of the interferometer is set reproducibly. The alignment workstation, shown in Fig. 4, comprises a telescope fitted with a video CCD to allow precise viewing of the various optical elements inside the interferometer, a white light source (tungsten lamp), a broadband green filter (centered around $540 \mathrm{~nm}$ ) illuminating a reticule R1 and a $50 \mathrm{~mm}$ SLR camera lens. The SLR lens projects an image of the reticule into the interferometer, 
usually at the output beamsplitter. In addition two alignment aids are used: a reticule R2 fitted into a custom fixture such that it can be placed precisely in the center of and adjacent to the active surfaces of the beam splitters within the interferometer; and, an aluminum disk with center markings that can be substituted into the mounts holding the end mirrors.

The main steps to align the interferometers are: (1) set the angles of all four elements in the interferometer precisely such that the optical axis is centered on the four elements; (2) match the path lengths of the two paths in the interferometer to within $1.5 \mu \mathrm{m}$; and, (3) install the delay etalon and translate the adjustable leg precisely to the offset required for the given delay element. We measure each etalon thickness $h$ to approximately $\pm 10 \mu \mathrm{m}$ uncertainty using both a micrometer and a phase-stepping white light interferometer. Using the known index of refraction $n=1.4607$ for UV grade fused silica at $532 \mathrm{~nm}$ we can compute the translation offset $d$ to approximately $\pm 3 \mu \mathrm{m}$ uncertainty. In practice the optical path delay is known to $\pm 10 \mu \mathrm{m}$ uncertainty ( $\pm 30 \mathrm{fs}$ ), limited primarily by the reproducibility of the translation stage. A more detailed description of steps (1) and (2) is given in the appendix.

\section{Fringe adjustments}

When perfectly aligned (parallel outpu beams) the Mach-Zehnder interferometer has a uniform output intensity: a single phase across the output. For the line-imaging application a fringe comb is imposed on the output by slightly tilting the output beam splitter, thus imposing a linear ramp in relative optical path difference across the output field. Doppler shifts in the light passing through the interferometer result in changes in the optical phase at the output, and these in turn appear as shifts in fringe position. The spatial frequency of this imposed pattern is arbitrary and is usually set to provide from 12-25 fringes across the output image (corresponding to a range of 8-16 spatial resolution elements per fringe).

We chose the Mach-Zehnder design with the image plane situated on the output beam splitter for several reasons. By separating the splitting and recombining of the two paths into two elements, our arrangement employs conveniently small and inexpensive $25.4 \mathrm{~mm}$ diameter optical elements. The arrangement is symmetrical, because the active surfaces of the beam splitters can be arranged to cancel out the additional delays introduced by the

beam splitter substrates (each path passes through the substrate once, which is not the 
case with the Michelson geometry), and this symmetry simplifies our path length zeroing procedures. By placing the image plane at the output beam splitter we decouple adjustments that optimize fringe contrast from adjustments that change fringe orientation and fringe spacing. All of these adjustments can be made through a wide range of settings not limited by the relatively small $20 \mathrm{~mm}$ clear aperture of optical elements in the system. Fringe contrast depends primarily on the degree to which the images projected through the two arms of the interferometer are overlapped. This is easily optimized by slight adjustments of one of the end mirrors in the interferometer. The fringe orientation and spacing are controlled by slight tilt adjustments of the output beam splitter; since the image is located precisely on the output beam splitter plane this adjustment does not affect the image position (i.e. overlap), and therefore does not affect the fringe contrast.

There is one obvious disadvantage of this design: for some alignment tasks we place a reticule fixture (identical to R2 in Fig. 4) on the beam splitter to provide an object that can be imaged onto the detectors (see below). By placing these alignment aids on this surface the beam splitter is susceptible to being scratched or contaminated; these defects will appear in the detected images, since they are in focus. However, we believe that this problem is minor, because these defects are usually much smaller (few $\mu \mathrm{m}$ ) than the spatial resolution at the streak camera detector plane $(\sim 100 \mu \mathrm{m})$, and are not apparent in the detector images. Since the interferometer elements are small, they are inexpensive and can be replaced easily if necessary.

\section{Alignment monitors}

Each interferometer produces two outputs. The main output channel is the beam that passes through the output beam splitter parallel to and in the same direction as the input beam. The second output is formed from the beams that exit the interferometer at an angle of approximately 9 degrees from the input direction. This output is effectively identical to the signal output and carries the same image (but with inverted fringes). For each interferometer the second output is reimaged onto the detector plane of a video CCD camera, using a 300 mm achromat (L8a and L8b).

The video CCD camera is used to view the target during alignment procedures prior to a target shot. The success of this arrangement depends on the accuracy of the registration 
of the video image relative to the image projected onto the streak slit. We check this registration by placing an alignment reticule at the output beam splitter plane of each interferometer and illuminating it with a low power CW alignment beam. By centering the two output images simultaneously on the video monitor and the streak camera slit we can ensure that the target images viewed in the monitor are accurately registered onto the streak camera. The images viewed in the monitors can be used to perform in situ interferometer fringe adjustments (fringe contrast, spacing and orientation).

\section{E. Timing and intensity fiducials}

In order to take the most advantage of the data available in the streak record (timing, velocity from fringe shift, and shock reflectance from intensity) two fiducials are imposed onto the edges of each data record, as shown in Fig. 5. The first is the standard OMEGA timing fiducial containing 8 timing pulses with $\sim 0.5 \mathrm{~ns}$ spacing (the pulse pattern and its timing are known very precisely and can be used for sweep rate calibration and time rgistration). The second is an intensity fiducial which records the intensity of the probe pulse that is delivered to the diagnostic from the probe laser during the shot. The intensity fiducial is extracted by collecting the probe light reflected from the injection beam splitter B1 using a $50 \mathrm{~mm}$ SLR lens (L9). This light is focused into a $100 \mu \mathrm{m}$ multi-mode fiber (same fiber type as the timing fiducial fiber) and delayed through approximately $12 \mathrm{~m}$ of optical fiber before it is extracted and injected into the slits of the streak cameras. This delay is designed to match the path delay of the probe to the target chamber and back. By comparing the reflected probe beam intensity to the intensity fiducial one can extract information about the reflectance of the target during the shot.

\section{TARGET ILLUMINATION}

\section{A. Probe laser}

VISAR experiments require a relatively high power single-frequency probe beam. The duration of most laser-driven shock experiments is a few ns to several tens of ns at most. After accounting for losses the power levels required to produce good exposure on a streak camera during a time window of a few ns can exceed $5 \mathrm{~kW}$. We have found a good choice for 
generating the probe beam to be an injection-seeded frequency-doubled Q-switched Nd:YAG laser source with $\sim 10$ ns pulse duration. The primary advantage of using a pulsed laser is that high output power is easily available, it is possible to adjust the probe beam intensity to the required levels by attenuating filters, and it is relatively easy to synchronize the Q-switch of the probe laser with OMEGA timing system.

The probe laser power delivered to the diagnostic table is approximately $30 \mathrm{~kW}$, or 200 $\mu \mathrm{J}$ per pulse in the $10 \mathrm{~ns}$ FWHM pulse. This power level is far lower than the peak power available from the laser, so most of the beam is attenuated prior to injection in the fiber. Using the resulting power level still requires approximately OD 1 - 1.5 attenuation to obtain non-saturating signal levels on most experiments. A filter wheel located at the fiber output (not shown in Fig. 2 is used to control the power injected into the system. Some targets are not highly reflecting, and the extra available power allows one to extract signals from low-reflecting target surfaces.

\section{B. Fiber-coupled illumination}

The probe laser light is delivered to the diagnostic station through a $1 \mathrm{~mm}$ core diameter, 0.16 numerical aperture multi-mode fused silica optical fiber. This fiber-coupled arrangement has two advantages. Firstly, it allows us to place the laser head and associated injection optics in a remote location (possibly a different room from the target area). Secondly, it is a convenient way of producing a spatially-smooth source distribution over a well-defined numerical aperture. Spatial mode-mixing through the long delivery path $(\sim 30 \mathrm{~m})$ of the multi-mode fiber results in the formation of a random speckle pattern on the output surface of the probe delivery fiber. On average the probe beam intensity is highly uniform across the output face of the fiber, and its angular distribution is also uniform within the solid angle defined by the numerical aperture of the fiber. On very small spatial scales $(\sim$ few $\mu \mathrm{m})$, the beam is highly modulated. The spatial scale of the speckles is similar to the resolution limit of the optical system, while feature sizes of interest in our experiments are usually $10-100$ times larger than this. The probe beam is collected and collimated by a $50 \mathrm{~mm}$ lens on the diagnostic station, after which it is injected into the optical path through the input beam splitter. The probe beam illuminates the target over an area $\sim 1 \mathrm{~mm}$ in diameter, which matches the diameter of the laser drive beams used in shock experiments. 
Many of our experiments observe reflecting shock fronts propagating through a transparent medium; these shocks behave as high quality specular reflectors. There is usually always some curvature on these shock fronts, typically ranging from a few $\mathrm{mm}$ to a few $\mathrm{cm}$ radius. These curvatures are small relative to the relevant scales of the laser driven shock experiments ( $\sim 0.8 \mathrm{~mm}$ spot diameter and $<100 \mu \mathrm{m}$ propagation distance), so from the point of view of the shock physics one-dimensional motion is well approximated. However, relative to the optical system an optical surface with a few mm radius has a large optical power (effective focal length much shorter than the focal length of the imaging optics), and can increase the divergence of the reflected beam significantly. This is the primary reason for combining the relatively fast $\mathrm{F} / 3.3$ collection optic with the multi-mode illumination: The multi-mode illumination beam fills the numerical aperture of the imaging and collection optic completely and thereby samples the target surface at all possible angles of incidence allowed by the numerical aperture of the optic over the full diameter of the illuminated region. This maximizes the probability of returning the signal through the optical train, even for specular shock fronts with significant curvature.

\section{Depth of field}

The relatively fast optic used for our experiments limits the depth of field over which the reflecting surface remains in sharp focus. For experiments during which the reflector travels beyond the range of sharp focus, the observed data will represent a spatially-averaged velocity. This situation must be taken into account when interpreting results from a given experiment.

For external surfaces the depth of field is limited to about $25 \mu \mathrm{m}$, as defined by the amount of defocus required to make the geometric blur spot comparable to the best focus point spread function. For reflections originating within a refracting sample, the cone angle of collected light internal to the sample is reduced, and so the effective depth if field is larger, by a factor related to the index of refraction of the sample, for example $40 \mu \mathrm{m}$ in quartz. While this depth of field is still relatively small, many measurements take place on axial scale lengths not much larger than this - usually in the range of 25 to $100 \mu \mathrm{m}$. For example shock wave impedance matching experiments employ step heights typically ranging from 10 to $40 \mu \mathrm{m}$, which is comparable to the depth of field. 


\section{VELOCITY SENSITIVITY}

The velocity sensitivity of VISAR systems is often quoted as the velocity-per-fringe, or VPF. For VISARs with glass delay elements it is given by $[17,36]$,

$$
\mathrm{VPF}_{0}=\frac{\lambda}{2 \tau(1+\delta)},
$$

where $\tau$ is given by equation (1) and the correction term $\delta$ accounts for dispersion in the etalon (at $532 \mathrm{~nm}$ in fused silica $\delta=0.0318$ ). This sensitivity formula applies for Doppler shifts observed from free surfaces moving in vacuum. For many experiments this situation is not the case, and additional corrections must be factored in depending on details of the experiment.

\section{A. Sample-dependent corrections}

When the reflection originates at an interface viewed through a transparent shocked window, an additional correction factor must be applied, leading to the modified VPF expression[16],

$$
\mathrm{VPF}_{w}=\operatorname{VPF}_{0}\left(1+\Delta \nu / \nu_{0}\right)^{-1}
$$

Here the term $\Delta \nu / \nu_{0}$ depends on the refractive index in the shock-compressed window. Correction terms have been empirically determined for several windows including fused silica[16, 37], LiF[38], sapphire[16, 37], alpha quartz[39] and PMMA[16].

When the reflected beam originates from a shock front in flight inside a transparent dielectric medium the Doppler shift depends on the refractive index in the sample, and the sensitivity becomes,

$$
\mathrm{VPF}_{s}=\mathrm{VPF}_{0} n_{s}^{-1}
$$

where the factor $n_{s}$ is the index of refraction of the unshocked material, just ahead of the shock front. It is straightforward to derive this sensitivity formula using optical path difference arguments. We have tested this sensitivity formula directly by tracking the shock front in a transparent medium as it travels across a known thickness of the sample medium (usually a known thickness of a transparent solid dielectric, or a known gap thickness of a transparent fluid sample bounded by transparent solid anvil). One can integrate the VISARdetermined velocity between the times of entry and exit of the shock in the sample; this gives 
quantitative measure of the sample thickess that can be compared against an independent thickness measurement. We find that, in general, the VISAR-determined thickness matches the thickness measured by other means to within measurement uncertainties, thus providing an experimental verification of senstivity formula of equation (4)[40].

Evidently knowledge of the refractive index of the sample is important to determine shock velocities. This situation also implies that any effects which can modify the optical properties of the sample material in the region ahead of the shock front may introduce additional apparent Doppler shifts that are unrelated to the shock velocity (e.g. x-ray photoionization or pre-heating). These possibilites have to be scrutinized on a case-by-case basis.

\section{B. Doppler corrections for a fast lens}

The F/3.3 lens used for target illumination and signal collection is a relatively fast optic, and the light interacting with the target is distributed over a range of angles, up to $\sim 9$ degrees incidence. It is well-known that the Doppler shift imparted to the reflected beam is proportional to $\cos \left(\theta_{i}\right)+\cos \left(\theta_{r}\right)$, where $\theta_{i}\left(\theta_{r}\right)$ is the angle of incidence (angle of reflection); however, the VISAR sensitivity formulas assume that the light interacts with the reflecting surface at normal incidence. In order to achieve high precision measurements it is therefore important develop a further correction that takes the angular distribution into account.

Fast optic corrections for VISAR experiments have been worked out previously by Sweatt and Crump[41], who found a linear correction to the VPF given by,

$$
\sigma_{s c}=\frac{\epsilon}{2}+\frac{\epsilon^{2}}{6(1-\epsilon)}
$$

where $\epsilon=(1-\cos \beta) / 2$, and $\mathrm{NA}=\sin \beta$ is the numerical aperture of the collection optic. (There is an additional non-linear term that is $\mathrm{O}\left(\epsilon^{4}\right)$, small enough to be neglected.) The analysis of Sweatt and Crump assumed that a collimated illumination beam interacted with a diffusely reflecting target surface in vacuum at normal incidence $\left(\theta_{i}=0\right)$ and was scattered according to a Lambertian distribution into the aperture of the collecting lens. The Sweatt and Crump correction to the $\mathrm{VPF}$ is $\mathrm{VPF} \rightarrow \mathrm{VPF}\left(1+\sigma_{s c}\right)$; for an $\mathrm{F} / 3.3(\mathrm{NA}=0.149)$ lens this correction is small: $\sigma_{s c}=0.003$.

Our experimental geometry differs from the assumptions of Sweatt and Crump in three 
important ways. Firstly the incident beam fills the collection lens over its full aperture, thus sampling the target with a uniform distribution over all possible angles of incidence allowed by the lens; secondly, the reflecting surface is a specular reflector in most cases; and, finally, the reflecting surface is often embedded within a refracting sample medium (a shock front in flight in the sample, or a reflecting interface behind a transparent window). The last difference means that the local angle of incidence at the reflecting surface is smaller than its external value owing to refraction, and the correction therefore depends on the index of refraction in the sample at the point where the reflection takes place. We have applied the method of analysis outlined by Sweatt and Crump to this new context and find that the appropriate formula for the Doppler error is to a very good approximation given by,

$$
\sigma_{F}=\epsilon_{F}+\frac{\epsilon_{F}^{2}}{3\left(1-\epsilon_{F}\right)}
$$

where $\epsilon_{F}=\left(1-\cos \left[\arcsin \left(\mathrm{NA} / n_{s}\right)\right]\right) / 2$, depends on the index of refraction $n_{s}$ at the reflecting surface as well as the numerical aperture NA of the collection optic. For $n_{s}=1$ (vacuum free surface) $\sigma_{F}=2 \sigma_{s c}$. It turns out that $\sigma_{F}$ is largely independent (to within $1 \%$ ) of the scattering properties of reflector (specular or diffuse Lambertian). Thus we find that a general expression that takes into account finite numerical aperture (fast lens) effects for our illumination geometry is

$$
\mathrm{VPF}_{F}=\operatorname{VPF}_{x}\left(1+\sigma_{F}\right)
$$

where $x$ indicates either $w$ or $s$, corresponding to the two cases corresponding to equations (3) and (4). The fast lens tends to reduce the apparent fringe count from its normal incidence value, and therefore the corrected VPF is slightly larger. The worst case is reflection at a vacuum interface $\left(n_{s}=1\right)$, for which $\sigma_{F}=0.006$. The magnitude of this correction is somewhat smaller than, but comparable to, our estimated measurement accuracy (about 1\% for a measurement at $\sim 7$ fringes, see below). Therefore this correction is of no consequence for most situations. However, we anticipate improved detection methods in the future (e.g. push-pull quadrature recording) in which case these corrections will become important as measurement accuracy improves. 


\section{DATA REDUCTION}

\section{A. Phase extraction}

The interferograms recorded in all of our experiments are analyzed to extract fringe phase as a function of space and time. Several methods have been proposed for extracting phase information from interferograms of this type, including fringe tracking and global analysis[42], an approximate push-pull quadrature reduction method[25], non-linear fitting[43] (which we used in early work) and the Fourier Transform Method (FTM) introduced by Takeda et al.[44], as well as variations on FTM developed by many others[45-47]. We have found that the most effective phase extraction method is a variation of FTM. There is an extensive literature on this technique already existing, so we will only briefly describe the steps we use to extract phase. The FTM begins by assuming that the recorded fringe intensity can be represented as a real-valued function,

$$
S(x, t)=B(x, t)+A(x, t) \cos \left(\phi(x, t)+2 \pi f_{0} x+\delta_{0}\right) .
$$

The term $2 \pi f_{0} x+\delta_{0}$ represents the linear phase ramp produced by the tilted output beam splitter of background fringe pattern: this can be thought of as a carrier wave with spatial frequency $f_{0}$ and arbitrary phase offset $\delta_{0}$. (For the purposes of this discussion the background pattern is assumed to maintain a constant fringe frequency $f_{0}$; in practice $f_{0}$ varies slightly over $x$ owing to imperfections in the interferometer optics, and distortions introduced by the streak camera.) The data is encapsulated in $\phi(x, t)$ which is a phase modulation superimposed on the carrier wave. The function $B(x, t)$ represents a slowly varying background intensity, or the average unmodulated signal intensity, while $A(x, t)$ represents the fringe amplitude, such that $|A| \leq B$. An equivalent representation of equation (8) is,

$$
S(x, t)=B(x, t)+C(x, t) \exp \left[2 \pi i f_{0} x+i \delta_{0}\right]+\text { c.c. }
$$

where $C(x, t)=A(x, t) \exp [i \phi(x, t)] / 2$. Taking a one-dimensional spatial Fourier transform at fixed time yields

$$
s(f, t)=b(f, t)+c\left(f-f_{0}, t\right)+c^{*}\left(f+f_{0}, t\right),
$$

which separates the background function $b(f, t)$ from the phase information contained in $c$. The FTM proceeds by choosing a filter that selects a broad range of the power spectrum 
around the $c$-lobe at positive frequencies and zeroes out the information at all other frequencies (including the negative $c^{*}$-lobe). Back transforming produces the complex-valued function,

$$
D(x, t)=C(x, t) \exp \left[2 \pi i f_{0} x+\delta_{0}\right]
$$

A "wrapped" phase can be extracted from this complex valued function,

$$
W\left(\phi(x, t)+2 \pi f_{0} x+\delta_{0}\right)=\arctan [\operatorname{Re}(D), \operatorname{Im}(D)]
$$

The wrapped phase function, $W$, is bounded in the interval $[-\pi, \pi]$, resulting in discontinuities when the phase passes through odd multiples of $\pi$. The final two steps are to unwrap the phase (remove the $2 \pi$ discontinuities), and to subtract the the background, i.e. $2 \pi f_{0} x+\delta_{0}$, leading to the extracted phase. A graphical illustration of this procedure showing the main steps is shown in Fig. 6.

Although the spatial resolution of the imaging system is $\sim 4 \mu \mathrm{m}$ the effective spatial resolution for velocity measurements is lower. This is easily seen within the context of the FTM because of the bandpass spatial filter that is applied to extract the fringe phase. As a consequence, the effective resolution depends on both the interferometer setting (number of fringes) and the filter band width used in the phase extraction process, both of which are under user control. A rough rule of thumb is that the number of independently resolvable spatial resolution elements available for velocity extraction is a few per fringe.

\section{B. Velocity measurement accuracy and detection limit}

We estimate that the uncertainty in the fringe phase is around \pm 0.05 fringe, or about \pm $0.3 \mathrm{rad}$. This is based on careful comparisons of reduced data extracted from simultaneous recordings taken at low and high sensitivities. The origin of this uncertainty is systematic, inherent to the recording method, and present independent of the method of phase extraction. This is because all data reduction techniques for extracting the phase from the fringe comb must assume that the background functions $A$ and $B$ are slowly varying in a spatial sense. In almost all practical situations this assumption is only approximately true because of the presence of high frequency spatial noise, for example from the speckle, and because of discontinuities in the spatial phase patterns for many types of data (e.g. across stepped 
target structures). Within the context of the FTM, there is always spectral content contributed by these various background and noise sources at frequencies close to the carrier frequency: this causes apparent mixing of phase and amplitude terms, and contaminates the extracted phase. Schemes have been proposed to improve this situation[48], but these require separate calibrations and assumptions that are incompatible with our experiments. The only way to reduce this uncertainty is to employ a more sophisticated data recording scheme (similar to phase-stepping techniques) in which multiple (at least three) phase-shifted versions of the interferogram are simultaneously recorded to allow algebraic cancellation of most of the background noise sources. The push-pull quadrature VISAR recording scheme of Hemsing[19] (a four-frame technique) has become the accepted standard VISAR recording method for discrete channel recording. Hemsing later demonstrated a line-imaging push-pull quadrature recording technique[21]; however, the scheme is significantly more complicated and difficult to implement than the single-frame method. It is conceivable that a push-pull quadrature recording scheme can reduce the phase extraction error by an order of magnitude. However, to the best of our knowledge no push-pull quadrature line-imaging VISAR system is being used routinely in a production environment, because of its complexity. We anticipate a probable need for it as measurement precision improves in the future.

The moderate accuracy of the phase extraction from the fringe pattern means that sensitive interferometer settings are needed to obtain high accuracy on Doppler shift measurements. For accurate measurements we choose settings such that one of the interferometers operates with a shift of around 7 fringes. With the systematic uncertainty of \pm 0.05 fringe a data record containing a shift of 7 fringes results in a relative uncertainty of $0.05 / 7<1 \%$ in the determination of the Doppler shift (velocity). Even with this strategy of large fringe counts, the phase extraction uncertainty remains the dominant contribution to the measurement uncertainty by a large factor (for example, the uncertainty in $\tau$ is less than $0.1 \%$ for a $7 \mathrm{~mm}$ etalon, about ten times smaller than the phase extraction uncertainty). The \pm 0.05 fringe uncertainty applied to the most sensitive VPF (30 mm etalon with $\mathrm{VPF}_{0}=1.7 \mathrm{~km} \mathrm{~s}^{-1}$ fringe $^{-1}$ ) indicates that the system detection limit is $\sim 0.09 \mathrm{~km} / \mathrm{s}$. 


\section{Resolution of fringe ambiguities}

With a few exceptions almost all of the data recorded with the line-VISAR system at OMEGA contain discontinuous fringe shifts. A common situation that produces such discontinuities is when a strong shock breaks through a motionless metal base plate into a transparent sample to produce a reflecting transmitted shock. The temporal resolution of the streak camera (about 10 - 50 ps depending on the sweep rate) is usually not fine enough to resolve the rapid fringe motion during the transition time $\tau$ of the interferometer (about 15 - $50 \mathrm{ps}$ ), so the recorded pattern is discontinuous. As noted above, the fringe discontinuities can amount to as many as 6 or 7 fringes plus a fractional part. For some kinds of experiments it may not be possible reliably to determine the integer part of the fringe shift from the data alone; this results in a fringe ambiguity. A reliable method to resolve fringe ambiguities is to record the data simultaneously on two separate interferometers with different sensitivities. This is the primary reason for the dual interferometer setup. With this arrangement analysis and comparison of the data sets from both channels will lead to a unique determination of the Doppler shift, and resulting velocity. Typically we choose the sensitivities such that the one channel is recording data between 2.5 and 3.5 fringes while the second channel records at a shift of between 6 and 8 fringes; this corresponds to a ratio

of sensitivities ranging from 2:1 to 2.5:1 (e.g. a typical configuration employs $7 \mathrm{~mm}$ and 3 mm etalons).

Fig. 7 illustrates the process of resolving the fringe ambiguities arising from a non-trivial sequence of discontinuities: a shock that is transmitted from an opaque pusher into a transparent fluid sample, reflected from a transparent anvil, then overtaken by a reverberation. Different fringe shifts must be applied at all of the discontinuities and different VPFs must be applied within the different media (sample and anvil) in order to reconstruct the velocity history. The top right panel in Fig. 7 shows the possible velocity histories that are consistent with the raw data for a sequence of 3 possible choices of fringe offsets for channel 2 (spaced by single fringes, dashed curves), and 5 choices for channel 1 (also spaced by single fringes, solid curves). In the absence of the information from the second interferometer a multitude of interpretations are possible, and one must introduce additional information to resolve the ambiguities. (Alternatively one can use a single interferometer with low sensitivity such that fringe shifts are $\sim 1$ fringe.) By combining the information from a dual interferometer setup 
no additional information is needed: matches between the two recordings are obtained for a unique set of integer fringe offsets, leading to a uniquely-determined velocity profile shown in the bottom right of the figure.

\section{SYSTEM OPERATION}

Operation of the ASBO system is aided by the fact that it makes available, through the video CCD cameras, near-real time images of the target as seen by the diagnostic during alignment and preshot operations. The probe laser sends out pulses at a $10 \mathrm{~Hz}$ rate, thus providing the experimenter and shot operations staff with an accurate indication of the alignment status of the target and the diagnostic at all times. The probe system is synchronized to the OMEGA master timing system so that one of the $10 \mathrm{~Hz}$ pulses is synchronous with the main laser shot. Prior to the main shot test triggers are generated routinely to allow the cameras to record test sweeps (dry run tests) of the returned signal. This excercises the entire system up to and including the data recording stage. The streak cameras employ computer controlled scientific grade CCD readouts, to allow quick viewing of the test sweeps prior to the shot. These capabilities allow signal levels, fringe quality, alignment status and trigger timing settings to be checked, adjusted and tested prior to each shot. The system is therefore robust and reliable; failures caused by misalignment, mistiming or poor signal quality are rare.

During the shot sequence the streak camera output detectors record three images: a background dark image (for subtracting CCD dark current background), a test image of the stationary fringe pattern streaked prior to the shot, and finally the shot data. The first two images are recorded during the countdown sequence a few seconds before the main shot. The stationary fringe recording contains the background phase $2 \pi f_{0}+\delta_{0}$, and is therefore useful for background subtraction. It also provides a signal intensity reference that can be used to help analyze the data recording for target reflectance.

Between shots the first element of the optical train (L1) is replaced, a task that results in slight shifts $(\sim 100-200 \mu \mathrm{m})$ of the pointing and focusing of the diagnostic. To adjust for this we usually place a reference grid at the location of the drive laser beams (which generate the pressure source for the shock), and align and focus the instrument to this reference. Following this step the experimental target is moved into the shot position. 


\section{EXPERIMENTS}

\section{A. Breakout measurements}

Fig. 8a shows an example of a step breakout measurement of a uniform shock front propagating through a sequence of steps on an opaque metallic target. Breakout measurements of this type are typically required for performing impedance match equation of state experiments on metals or opaque materials at high pressures (> few x $100 \mathrm{GPa}$ ). Luminescence detection can provide equivalent information if the shock is strong enough; however, the reflection mode of detection is superior because the initial signal level can be set accurately near to the saturation level of the detector, and provide the maximum possible dynamic range. The breakout time is accurately indicated because the reflection is extinguished to undetectable levels in a very short time interval, about 10 ps. This is because the sample expands as an ionized plasma; once the profile has expanded to a scale length comparable to the probe wavelength, the reflectivity drops exponentially. (Reflections are not extinguished if the surface remains solid after the shock release; however, that motion can be detected as a fringe shift, described in the next section.)

The primary observables in this example are the breakout times, and the presence of the fringes is not necessary for the measurement. (They can be removed by blocking one arm of the interferometer.) However the velocity interferometer does provide secondary information if is set sensitively enough to detect slight motions of the surface prior to the shock breakout. Such motion would indicate the presence of preheat, and is of concern to the experimenter. In this particular example no fringe motion is evident, indicating low or negligible preheat levels.

As a second example of breakout measurements, Fig. 8b shows the breakout patterns of a modulated shock passing through a uniform foil target. In this target a $0.5 \mu \mathrm{m}$ thick Al layer was embedded in the middle of a $20 \mu \mathrm{m}$ thick polystyrene foil and the target was driven by a laser drive that was spatially modulated with a sinusoidal intensity variation of approximately $60 \mu \mathrm{m}$ period. Breakout through the Al layer is seen first around $1 \mathrm{~ns}$ when the reflected intensity is partially extinguished, followed by breakout out through the rear surface of the foil around $2 \mathrm{~ns}$, when the reflection is fully extinguished. The variation in breakout times can be correlated directly to the spatially modulated pressure profile of 
the drive. In order to observe the modulated breakout clearly the fringes were removed by blocking one arm of the interferometer. The high frequency spatial intensity modulations

are caused by the speckle distribution in the illumination beam. Further details of these experiments can be found in[49].

Accuracy of breakout measurements is limited by the performance of the streak camera, and the accuracy of the sweep calibration. This varies depending on the settings of the camera; at the fastest sweep rates over a few ns temporal window we achieve around \pm 20 ps.

\section{B. Free surface and interface motion}

The VISAR is especially useful for measuring the motion induced by low stresses in the elastic-plastic reponse range of many materials. These stresses usually leave the sample in the solid phase most of the time, at relatively low temperatures (few $100 \mathrm{~K}$ ). These experiments generate temperatures that are undetectable with luminescense techniques, but are well-suited to velocimetry because high surface reflectivities are maintained.

Fig. 9 shows a recording of the motion of a thin stepped $\mathrm{Al}$ foil driven by a shockless (slowly rising) pressure pulse. The thin step $(8.1 \mu \mathrm{m})$ of the foil accelerates smoothly and continuously, while the thicker part $(18.4 \mu \mathrm{m})$ of the foil shows a later and slower acceleration. The observed accelerations correspond to peak stresses around $200 \mathrm{GPa}$ (2 Mbar) in the foil. These velocity measurements are essential for characterizing the pressure pulse driving the motion. For shockless drives these measurements may be used quantitatively to extract isentropic compression curves for the target material. This type of measurement cannot be carried out with any other diagnostic (e.g. luminescence detection, or radiography) to the same level of precision and detail.

\section{Impedance matching equation of state in transparent samples}

An important class of laser-driven shock experiments that has been enabled by the lineVISAR at OMEGA is the measurement of the equation of state (EOS) of transparent dielectric samples through impedance matching. The objective of such experiments is to determine the Hugoniot equation of state generated in a sample by transmitting a shock wave from 
a standard reference material into the sample material. The Hugoniot state in the sample can then be determined in a relative fashion through the known EOS of the reference material. Two primary observables are needed: (1) the shock velocity in the reference material; and, (2) the shock velocity in the sample. The basic configuration if these experiments is shown in Fig. 10. In most of our experiments we use a stepped Al baseplate as the reference material: by measuring both the height of the $\mathrm{Al}$ step, and the breakout times across the step the shock speed in the reference can be determined. The VISAR fringe shift is used to determine the shock velocity in the sample: this measurement presumes that the shock is strong enough to ionize the sample into a reflecting state in order for a returned signal to be observed.

The VISAR technique provides a key advantage over step breakout measurements because it produces a continuous record of the shock velocity history. This is important because it provides an immediate assessment of the steadiness of the shock propagation in the sample. In this case shown in Fig. 10 the shock is steady during the measurement time, and begins to attenuate in the sample after $t=6 \mathrm{~ns}$. We have carried out such experiments in a number of dielectrics, including water, $\mathrm{LiF}$, sapphire, diamond, polystyrene, fused silica and alphaquartz. Threshold pressures required to obtain high shock reflectivities vary widely among these materials; in most cases the pressures are too high to be accessible with conventional shock drivers (explosives and gas guns) but are well within the capabilities of large laser facilities such as OMEGA.

Measurement accuracy of this technique depends on the accuracies of both the step breakout method and the VISAR phase extraction. As noted above the velocity uncertainty

of about $1 \%$ is of moderate accuracy, but it still exceeds the accuracies attainable for the step breakout measurement (about 2\% - 3\% for the conditions allowed by OMEGA). Because of this the step breakout measurement is the primary limiter on the accuracy of the impedance match EOS measurements at OMEGA.

\section{Reflected shocks}

For transparent materials the VISAR is particularly useful for obtaining precise measurements of reflected shock events using transparent anvils. For these experiments a shock is transmitted from a low impedance fluid into a higher impedance anvil; the incident shock 
resolves into a transmitted and a reflected shock. In the example shown in Fig. 11 the impact occurs at at $\sim 4.8$ ns: just prior to this time the shock velocity in the sample is 25.2 $\mathrm{km} / \mathrm{s}$ and the shock transmitted into the anvil has velocity $21.8 \mathrm{~km} / \mathrm{s}$.

By measuring the shock velocity in the sample fluid and the transmitted shock state in the anvil the double shock state in the fluid can be determined as long as the principal Hugoniots are known in both materials. The line-VISAR technique has an advantage over conventional methods based on transit time measurements because the velocity measurement is localized both spatially and temporally, thus minimizing systematic errors associated with non-steady and/or non-planar propagation. We have carried out such experiments using water and liquid deuterium as sample fluids and alpha-quartz, fused silica, sapphire and LiF as anvils.

\section{E. Reflectivity of attenuating shocks}

The line-imaging VISAR can provide valuable information on transport properties in shocked fluids because the fringe pattern encodes both the reflectivity and the velocity of the shock front. From this pair of observables it is possible to construct a quantitative relationship between the reflectance and shock velocity over a wide range of velocities in a single experiment. These experiments use non-steady attenuating shocks to sample the range of states. Even though the shock is non-steady the material at the leading edge of the shock front is always compressed to a state on the principal Hugoniot. The reflection originates within a skin depth of the metallic fluid, a distance of the order of $100 \mathrm{~nm}$ or less, over which the thermodynamic variables vary only slightly. Therefore the observed reflectance represents a continuous sampling of states distributed at or very near to the principal Hugoniot through a wide range of pressures. This information is of value for inferring the variation of electrical conductivity and carrier density along the Hugoniot. This technique has been demonstrated in deuterium[28], as well as LiF and sapphire[29].

Fig. 12 shows an example of an attenuating shock in diamond. The shock was produced by driving a target comprised of a $20 \mu \mathrm{m}$ polystyrene ablator, a $50 \mu \mathrm{m} \mathrm{Al}$ baseplate and $\mathrm{a} \sim 250 \mu \mathrm{m}$ thick diamond plate. The target was driven by a $1 \mathrm{~kJ}$ pulse of 1 ns duration. X-rays generated in the laser plasma cause a brief period of photoionization in the diamond, rendering it partially opaque around $t=1 \mathrm{~ns}$. The X-ray source terminates with the end of the laser pulse and the diamond sample becomes transparent again (through recombination 
of the photoionized electrons): the fringe pattern reappears unshifted at $\sim 1.5 \mathrm{~ns}[50]$. The breakout from the baseplate at $\sim 2.6 \mathrm{~ns}$ is simultaneous across the $650 \mu \mathrm{m}$ spot, indicating that the shock is initially planar; however, it is attenuating because the applied pressure pulse is brief, and rarefaction waves rapidly overtake the leading shock front before it breaks out of the Al baseplate. Initially, the fringes are visible over the full diameter of the laser spot $(\sim 650 \mu \mathrm{m})$. Later, edge rarefactions move towards the central axis causing loss of signal beyond the edges. The shock remains flat in the central $200 \mu \mathrm{m}$ for the duration of the recording. Here the reduction in the signal is related to the intrinsic reflectivity of the shock front.

Around 20 ns the shock emerges from the rear surface. The curvature on the shock front is evident from the variation in breakout time across the diameter. The persistence of the reflection for a few ns after the breakout indicates that the rear surface of the sample has released into the solid phase, thus retaining a sharp reflecting interface.

During this experiment the shock velocity initially launched in the diamond sample is $\sim$ $40 \mathrm{~km} / \mathrm{s}$ corresponding to $\sim 3900 \mathrm{GPa}(39 \mathrm{Mbar})$ pressure, and decays to $\sim 20.5 \mathrm{~km} / \mathrm{s}, \sim$ 600 GPa (6 Mbar). Corresponding temperatures and densities (estimated from theoretical EOS models) vary between $90000 \mathrm{~K}$ and $5000 \mathrm{~K}$, and $10 \mathrm{~g} \mathrm{~cm}^{-3}$ and $6 \mathrm{~g} \mathrm{~cm}^{-3}$, respectively. The observed reflectivity is that of a dense strongly-coupled carbon plasma.

\section{F. Applications to inertial confinement fusion}

Inertial confignement fusion (ICF) requires the control and timing of a series of shocks launched by a complex stepped driving pulse. For example, a shock timing scheme for the indirect drive form of ICF requires a sequence of three shocks to coalesce nearly simultaneously at a prescribed depth within the target fuel[51]. A line-imaging VISAR will be used for experiments to observe these shocks and verify their relative timing behavior.

Fig. 13 shows an example of an experiment designed to approximate a pulse delivery schemes for direct drive ICF. In this example two attenuating shocks are launched into a target (polystyrene in this case), by applying a sequence of two brief intense laser pulses. X-rays generated by the surface plasma created by the first pulse causes a brief period of photoionization in the target, which renders it partially opaque; it gradually relaxes to become more transparent, and the VISAR signal reveals an attenuating shock propagating 
through the target after about 0.4 ns. Between 0.4 and 4 ns the fringe pattern shows a strong continuous deceleration of the shock as would be expected. The second laser drive pulse at 2 ns causes another loss of signal intensity (but the fringe phase remains continuous). The fringe discontinuity associated with this second shock is seen later at 4 ns when the second shock overtakes the first. The fringe pattern terminates at $6 \mathrm{~ns}$ when the shock breaks through the rear surface of the plastic target. Detailed comparison of these data with numerical models of the laser-target interactions will aid greatly in understanding the coupling of the laser to the target for these short intense pulses.

\section{SUMMARY AND CONCLUSION}

In summary we have described in detail the configuration and operation of the LLE ASBO diagnostic, a line-imaging VISAR that is routinely used at the OMEGA laser facility. The instrument can measure velocities ranging from 0.1 to more than $50 \mathrm{~km} / \mathrm{s}$ with $\pm 1 \%$ uncertainty over most of this range. It can measure spatially-resolved breakout patterns at temporal resolutions as low as 20 ps, with spatial resolutions $\sim 4 \mu \mathrm{m}$. Finally, it can produce quantitative reflectance data simultaneous with the velocity measurements.

The system described here has evolved out of experience with similar implementations fielded at various facilities including NOVA, Vulcan and Phebus. At OMEGA and these other facilities we have implemented variations and additions to the basic arrangement that are not included in the description above. Four of the most useful variations include: (1) schemes to lengthen the duration of the probe pulse; (2) schemes for simultaneous dual wavelength operation, for example at the fundamental (1064 nm) and second harmonic (532 $\mathrm{nm}$ ) of the probe laser; (3) the addition of an optical recording channel using the existing imaging relay to record thermal luminescence data simultaneous with the VISAR data; and, (4) the addition of dove prisms to enable arbitrary image orientation on the detector.

A line-imaging VISAR capability is currently being developed for the National Ignition Facility. Its design differs from the OMEGA implementation in many details, but bene-

fited greatly by the experience gained with the VISAR implementation on OMEGA and its predecessors. 


\section{Acknowledgments}

We wish to thank P. Bell, R. Costa, J. Cox and K. Haney of LLNL and G. Pien of LLE for help during the fabrication and installation of the instrument, and D. Kalantar and K.T. Lorenz for providing the data in Fig. 9, and J. Eggert for useful discussions. In addition we thank operations staffs of both the NOVA and the OMEGA laser facilities. This work was performed under the auspices of the U.S. Department of Energy by Lawrence Livermore National Laboratory under contract number W-7405-ENG-48, and supported by the U.S. Department of Energy Office of Inertial Confinement Fusion under Cooerative agreement No. DE-FC03-92SF19460, the University of Rochester, and the New York State Energy Research and Development Authority.

\section{APPENDIX A: INTERFEROMETER ALIGNMENT}

Following is the detailed procedure to align the interferometer. Using a plastic ruler, and with the delay etalon removed, the end mirror on the adjustable leg of the interferometer is set to a position to match the path of the fixed leg within the accuracy of the ruler

measurement (usually within $0.5 \mathrm{~mm}$.) (1) The telescope line of sight is adjusted to view precisely through the aperture centers of IB2 and IM2 on the interferometer (see Fig. 3 and Fig. 2); this procedure is accomplished with the help of alignment aids placed at the IB2 and IM2 positions. (2) The angle of the beam splitter IB2 is adjusted to center the aperture of IM1 as viewed through the telescope. (3) With reticule R2 placed at IB1 and the telescope focused on it the angles of IM1 and IM2 are adjusted to center the double image of R2 as viewed through both paths. (4) R2 is removed, the telescope is focused to the IB2 plane, and an image of reticule R1 is projected into the interferometer and also focused at the IB2 plane, where a double image is observed. (5) The angle of IB1 is adjusted to cause precise overlap of the double image at IB2. (6) Relative path difference is adjusted by translating the motorized mirror (no etalon) slowly (velocity $<2 \mu \mathrm{m} / \mathrm{s}$ ) until white light fringes appear in the image at the beam splitter plane IB2. The paths are matched by translating until the central fringe of the white light pattern is situated in the center of the image viewed by the telescope. Since each fringe represents approximately $0.5 \mu \mathrm{m}$ optical path difference, we estimate that the accuracy of matching the paths using this technique is $\sim 1.5 \mu \mathrm{m}$ (assuming 
the final fringe pattern is shifted by no more than 2 or 3 fringes away from the central fringe).

[1] L. Veeser and J. Solem, Phys. Rev. Lett. 40, 1390 (1978).

[2] R. Trainor, J. Shaner, J. Auerbach, and N. Holmes, Phys. Rev. Lett. 42, 1154 (1979).

[3] F. Cottet, J. P. Romain, R. Fabbro, and B. Faral, Phys. Rev. Lett. 52, 1884 (1984).

[4] D. Parfeniuk, A. Ng, and L. Dasilva, Optics Commun. 53, 389 (1985).

[5] T. Lower, R. Sigel, K. Eidmann, I. B. Foldes, S. Huller, J. Massen, G. D. Tsakiris, S. Witkowski, W. Preuss, H. Nishimura, et al., Phys. Rev. Lett. 72, 3186 (1994).

[6] P. Celliers, A. Ng, G. Xu, and A. Forsman, Phys. Rev. Lett. 68, 2305 (1992).

[7] A. Benuzzi, T. Lower, M. Koenig, B. Faral, D. Batani, D. Beretta, C. Danson, and D. Pepler, Phys. Rev. E. 54, 2162 (1996).

[8] R. Kauffman et al., Rev. Sci. Instr. 66, 678 (1995).

[9] J. Oertel et al., Rev. Sci. Instr. 70, 803 (1999).

[10] A. Ng, D. Parfeniuk, P. Celliers, and L. DaSilva, in Shock Waves in Condensed Matter, edited by Y. Gupta (Plenum, 1985), pp. 255-60.

[11] D. Parfeniuk, A. Ng, L. DaSilva, and P. Celliers, Optics Commun. 56, 425 (1986).

[12] A. Ng, D. Parfeniuk, P. Celliers, L. DaSilva, R. More, and Y. Lee, Phys. Rev. Lett. 57, 1595 (1986).

[13] M. Basko, T. Lower, V. N. Kondrashov, A. Kendl, R. Sigel, and J. Meyer-ter Vehn, Phys. Rev. E 56, 1019 (1997).

[14] T. Lower, V. N. Kondrashov, M. Basko, A. Kendl, J. Meyer-ter Vehn, R. Sigel, and A. Ng, Phys. Rev. Lett. 80, 4000 (1998).

[15] L. Barker and R. Hollenbach, Rev. Sci. Instr. 36, 1617 (1965).

[16] L. Barker and R. Hollenbach, J. Appl. Phys. 41, 4208 (1970).

[17] L. Barker and R. Hollenbach, J. Appl. Phys. 43, 4669 (1972).

[18] D. Goosman, J. Appl. Phys. 46, 3516 (1975).

[19] W. Hemsing, Rev. Sci. Instr. 50, 73 (1979).

[20] D. Bloomquist and S. Sheffield, J. Appl. Phys. 54, 1717 (1983).

[21] W. Hemsing, A. Mathews, R. Warnes, M. George, and G. Whittemore, in Shock Compression of Condensed Matter - 1991, edited by S. Schmidt, R. Dick, J. Forbes, and D. Tasker (Elsevier, 
1992), pp. $767-70$.

[22] K. Baumung, J. Singer, S. Razorenov, and A. Utkin, in Shock Compression of Condensed Matter - 1995, edited by S. Schmidt and W. Tao (AIP Press, Woodbury, NY, 1996), p. 1015.

[23] W. M. Trott and J. R. Asay, in Shock Compression of Condensed Matter - 1997, edited by S. Schmidt, D. Dandekar, and J. Forbes (AIP Press, Woodbury, NY, 1998), p. 837.

[24] P. Celliers, G. Collins, L. DaSilva, D. Gold, and R. Cauble, Appl. Phys. Lett. 73, 1320 (1998).

[25] W. M. Trott, M. D. Knudson, L. C. Chhabildas, and J. R. Asay, in Shock Compression of Condensed Matter - 1999, edited by M. D. Furnish, L. C. Chhabildas, and R. S. Hixson (AIP Press, Melville, NY, 2000), p. 993.

[26] E. Moshe, E. Dekel, Z. Henis, and S. Eliezer, Appl. Phys. Lett. 69, 1379 (1996).

[27] D. M. Gold, P. M. Celliers, G. W. Collins, K. S. Budil, R. Cauble, L. B. da Silva, M. E. Foord, R. E. Stewart, R. J. Wallace, and D. Young, Ap. J. Suppl. 127, 333 (2000).

[28] P. M. Celliers, G. W. Collins, L. B. Da Silva, D. M. Gold, R. Cauble, R. J. Wallace, M. E. Foord, and B. A. Hammel, Phys. Rev. Lett. 84, 5564 (2000).

[29] D. Hicks, P. Celliers, G. Collins, J. Eggert, and S. Moon, Phys. Rev. Lett. 91, 035502 (2003).

[30] P. Loubeyre et al. (2003), to be published.

[31] D. Batani, A. Morelli, M. Tomasini, A. Benuzzi-Mounaix, F. Philippe, M. Koenig, B. Marchet, I. Masclet, M. Rabec, C. Reverdin, et al., Phys. Rev. Lett. 88, 235502/1 (2002).

[32] E. Henry, D. Batani, M. Koenig, A. Benuzzi, I. Masclet, B. Marchet, M. Rebec, C. Reverdin, P. Celliers, L. Da Silva, et al., Laser Part. Beams 19, 111 (2001).

[33] A. Benuzzi-Mounaix, M. Koenig, G. Huser, B. Faral, D. Batani, E. Henry, M. Tomasini, B. Marchet, T. A. Hall, M. Boustie, et al., Phys. Plasmas 9, 2466 (2002).

[34] Equation (1) is approximate because the beam passes through the etalon slightly obliquely at $\theta=4.5$ degrees angle of incidence. With oblique incidence and using the usual translation offset, $d=h(1-1 / n)$, it can be shown that the exact expression for the delay is $\tau^{*}(\theta)=$ $(2 h / c)[n / \cos \phi-1 /(n \cos \theta)]$, where $n \sin \phi=\sin \theta$. A Taylor series expansion in $\theta$ reveals that $\Delta \tau / \tau=\left(\tau^{*}(\theta)-\tau\right) / \tau \sim \mathrm{O}\left(\theta^{4}\right)$ and $|\Delta \tau / \tau|<10^{-5}$ for our interferometer. Since our relative measurement uncertainty is $\sim 1 \%$, equation (1) is accurate enough for our purposes.

[35] Ideally the required delay should be accomplished with a single element; if multiple elements are used it is advisable to use spacers to maintain an air gap $>1 \mathrm{~mm}$ between adjacent elements, in order to avoid thin film interference effects causing reflections at the interface. 
[36] L. Barker and K. Schuler, J. Appl. Phys. 45, 3692 (1974).

[37] R. Setchell, J. Appl. Phys. 50, 8186 (1979).

[38] J. Wise and L. Chhabildas, in Shock Compression of Condensed Matter - 1985, edited by Y. Gupta (Plenum, 1986), p. 441.

[39] S. Jones and Y. Gupta, J. Appl. Phys. 88, 5671 (2000).

[40] This sensitivity formula has been independently confirmed by measurements of shock propagation in liquid deuterium by M.D. Knudson, J.R. Asay and others of Sandia National Laboratory, private communication M.D. Knudson.

[41] W. Sweatt and O. Crump Jr., Rev. Sci. Instrum. 62, 2946 (1991).

[42] G. Fisk, G. Mastin, and S. Sheffield, J. Appl. Phys. 60, 2266 (1986).

[43] J. Slepicka and S. Cha, Appl. Opt. 34, 5039 (1995).

[44] M. Takeda, H. Ina, and S. Kobayashi, J. Opt. Soc. Am. 72, 156 (1982).

[45] W. J. Macy, Appl. Opt. 22, 3898 (1983).

[46] K. Nugent, Appl. Opt. 24, 3101 (1985).

[47] C. Roddier and F. Roddier, Appl. Opt. 26, 1668 (1987).

[48] J. Lui and P. Ronney, Appl. Opt. 36, 6231 (1997).

[49] T. Boehly, T. Collins, O. Gotchev, T. Kessler, J. Knauer, T. Sangster, and D. Meyerhofer, J. Appl. Phys. 92, 1212 (2003).

[50] Photoionization effects in transparent samples can be suppressed with more sophisticated target designs that employ X-ray-attenuating layers containing high-Z materials, for example plastics doped with a few atomic \% of Br or I.

[51] D. Munro, P. Celliers, G. Collins, D. Gold, L. Da Silva, S. Haan, R. Cauble, B. Hammel, and W. Hsing, Phys. Plasmas 8, 2245 (2001).

This work was performed under the auspices of the U.S. Department of Energy by University of California, Lawrence Livermore National Laboratory under Contract W-7405-Eng-48. 


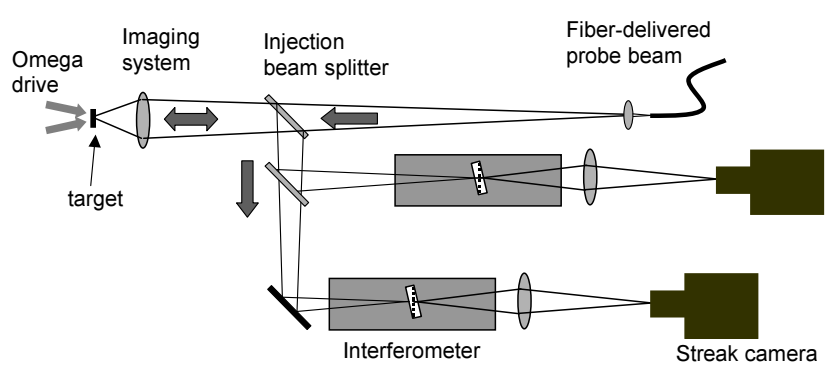

FIG. 1: Conceptual layout of the OMEGA line-imaging shock breakout diagnostic. An image of the target is relayed to the streak cameras through a pair velocity interferometers. 


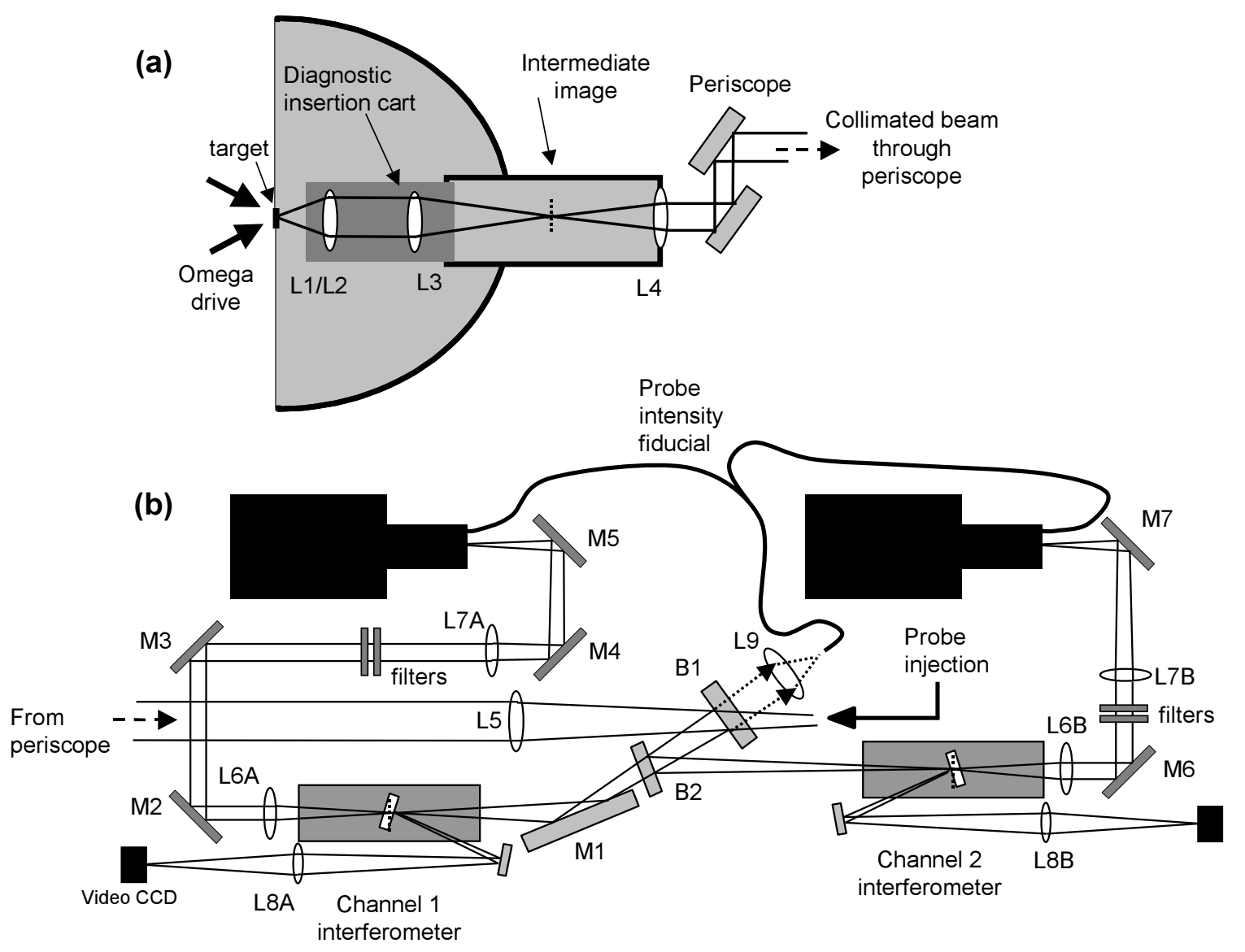

FIG. 2: Details of the image relay system. (a) The front section is a cascade of two image relays with an intermediate image plane localized between L3 and L4. (b) The output image is formed by L5 inside the interferometers, and relayed to the streak cameras through the L6 and L7 pairs of lenses. The probe beam is injected through B1, and the signal path is split into the two channels by B2. (Timing fiducial fibers are not shown.) 


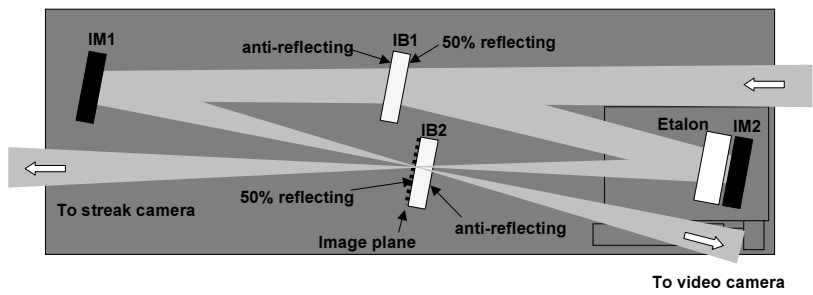

FIG. 3: Detail of the interferometer design. The incoming beam focuses to form an image at the reflecting surface of the output beam splitter, IB2. Slight tilts of IB2 change the fringe orientation and fringe spacing without affecting contrast. Slight adjustments of the tilt of one of the end mirrors adjust the fringe contrast (image overlap). 


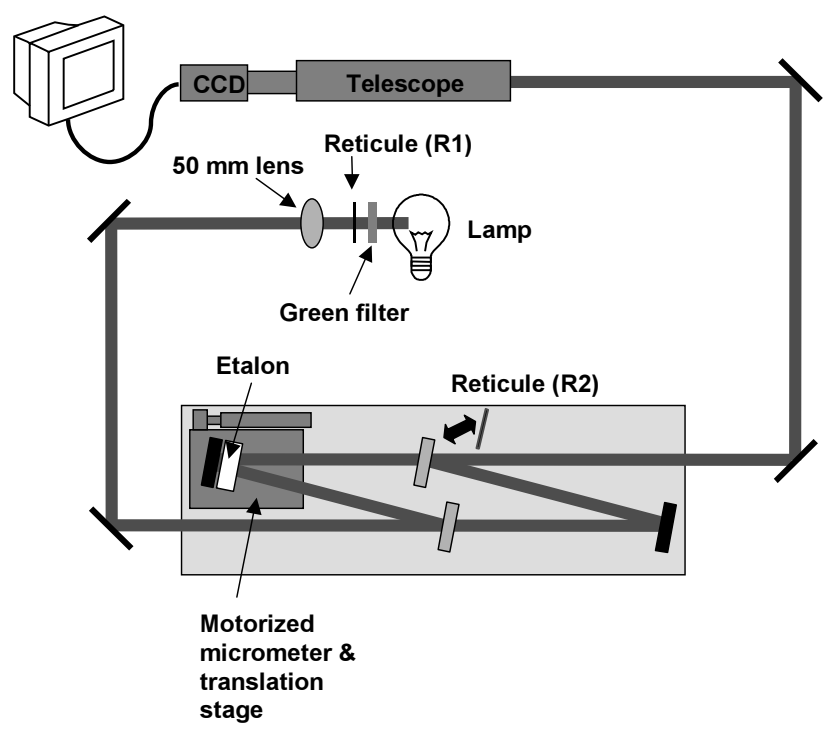

FIG. 4: Arrangement used for interferometer alignment. The telescope is used to view precisely through the centers of all the apertures in the interferometer, and to detect the presence of white light fringes localized at the reflecting surface of the output beam splitter when the path lengths of the two arms are equalized. 


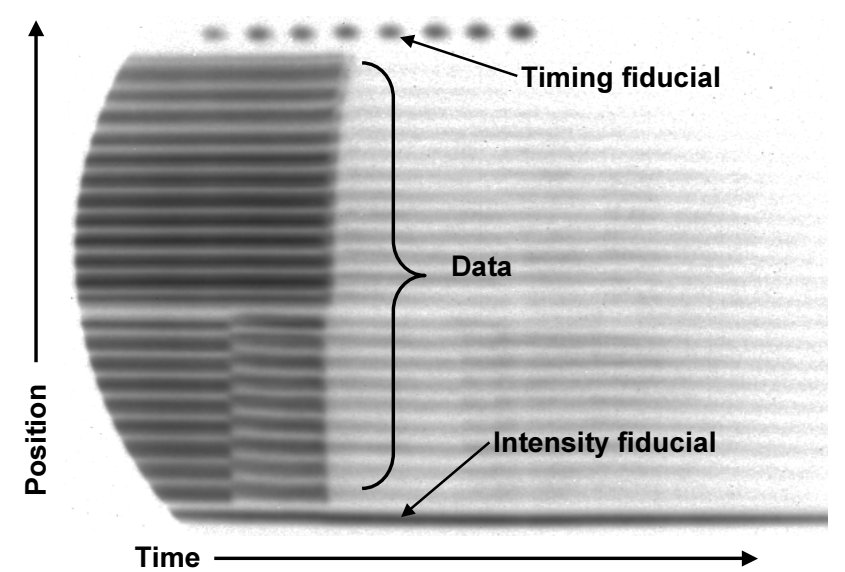

FIG. 5: Arrangement of fiducial traces recorded on the edges of a data record. The fiducial signals are injected at the cathode slit using a pair of $100 \mu \mathrm{m}$ core diameter optical fibers. 


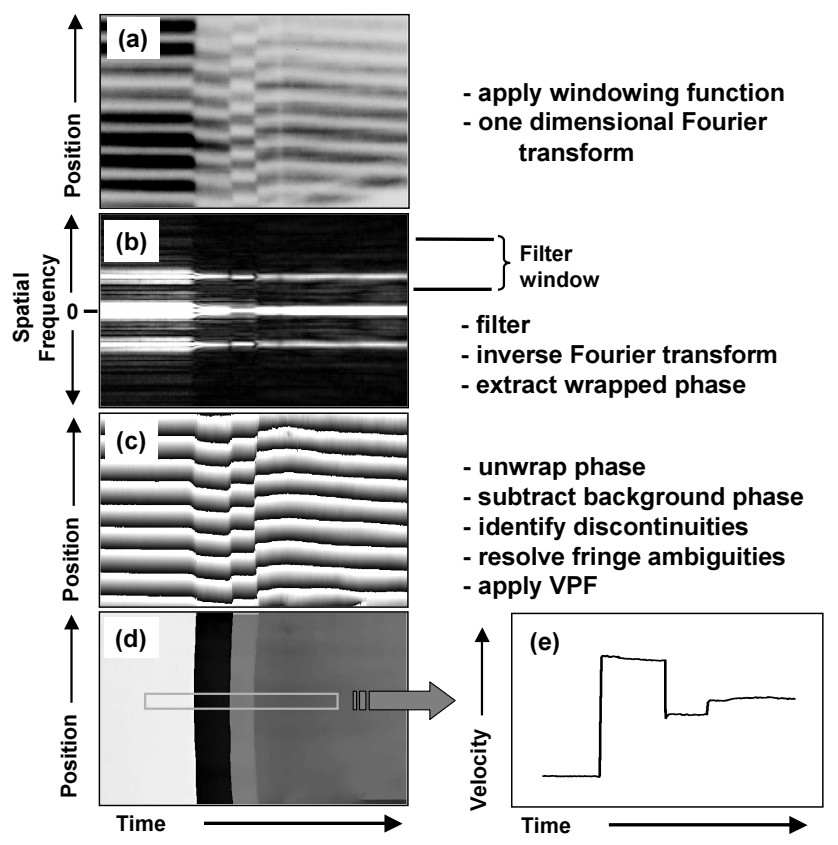

FIG. 6: Main steps if the phase extraction procedure using the Fourier Transform Method (FTM), details are explained in the text. Shown in the panels are (a) the raw data; (b) the discrete fourier spectrum; (c) the wrapped phase function, $W$ (equation (12)); (d) the final velocity field; and, (e) a lineout extracted from the velocity field. 


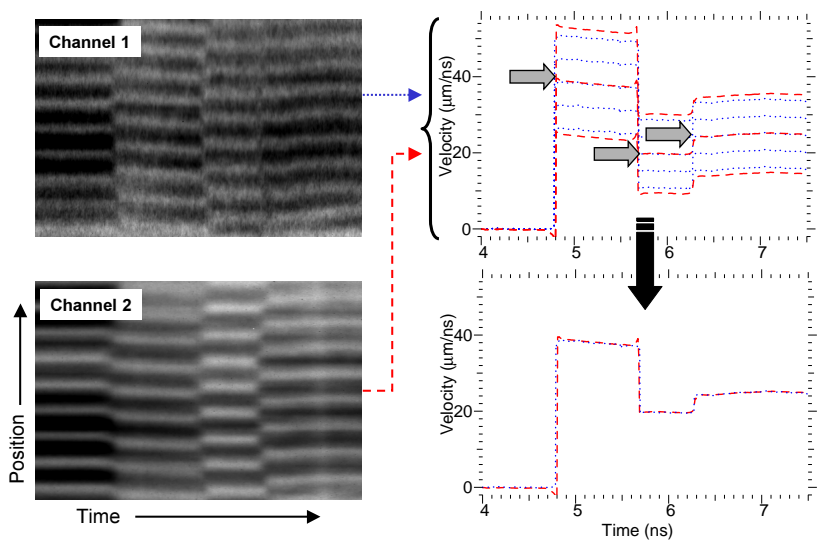

FIG. 7: Illustration of the resolution of fringe ambiguities. Channel 1 was operating with a $7 \mathrm{~mm}$ etalon $\left(\mathrm{VPF}_{0}=6.88 \mathrm{~km} \mathrm{~s}^{-1}\right.$ fringe $\left.{ }^{-1}\right)$ and channel 2 with a $3 \mathrm{~mm}$ etalon $\left(\mathrm{VPF}_{0}=15.96 \mathrm{~km} \mathrm{~s}^{-1}\right.$ fringe $\left.^{-1}\right)$. The analyzed velocity profiles show a multitude of possible interpretations of the data based on one or the other of the two interferometers. Only one unique selection of possible fringe shifts produces close agreement for the Doppler shifts extracted from the two recordings. 

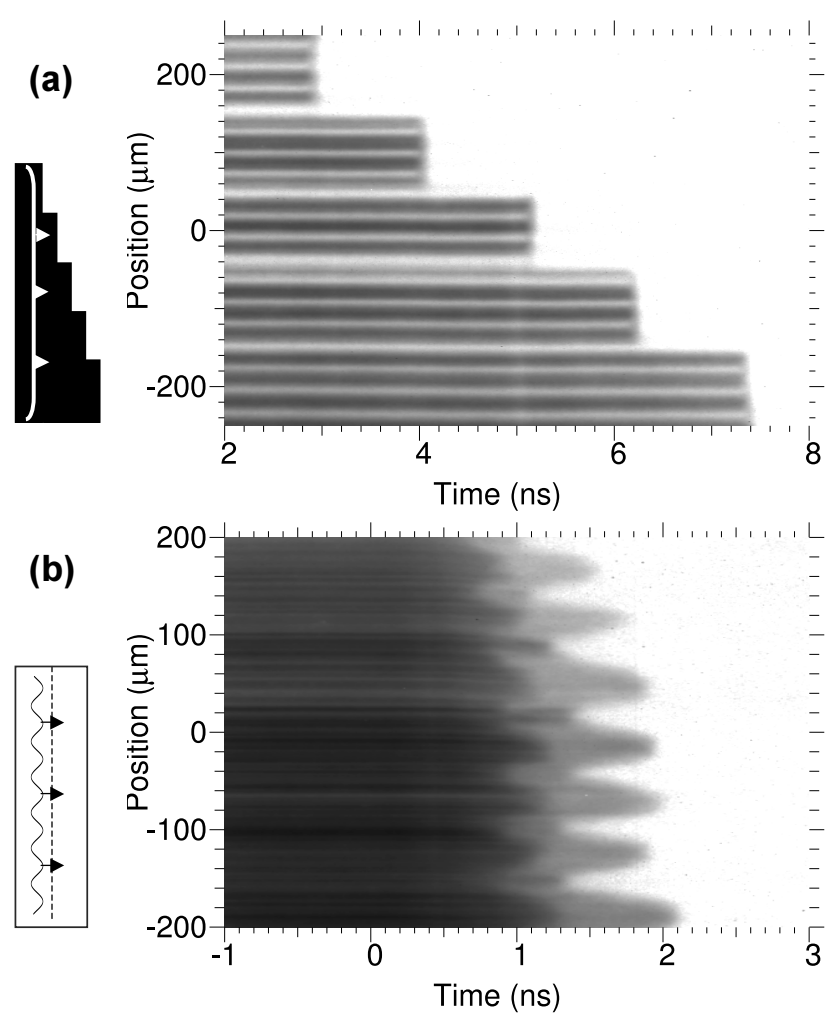

FIG. 8: (a) Shock breakout from a series of steps in an opaque metal target. Lack of fringe motions indicates the absence of preheat effects during this experiment. Shock velocity can be determined from the breakout times and the known step heights. (b) Shock breakout events in $20 \mu \mathrm{m}$ thick plastic foil with an embedded Al layer driven by a modulated pressure drive. The fringes were removed by blocking one arm of the interferometer. 


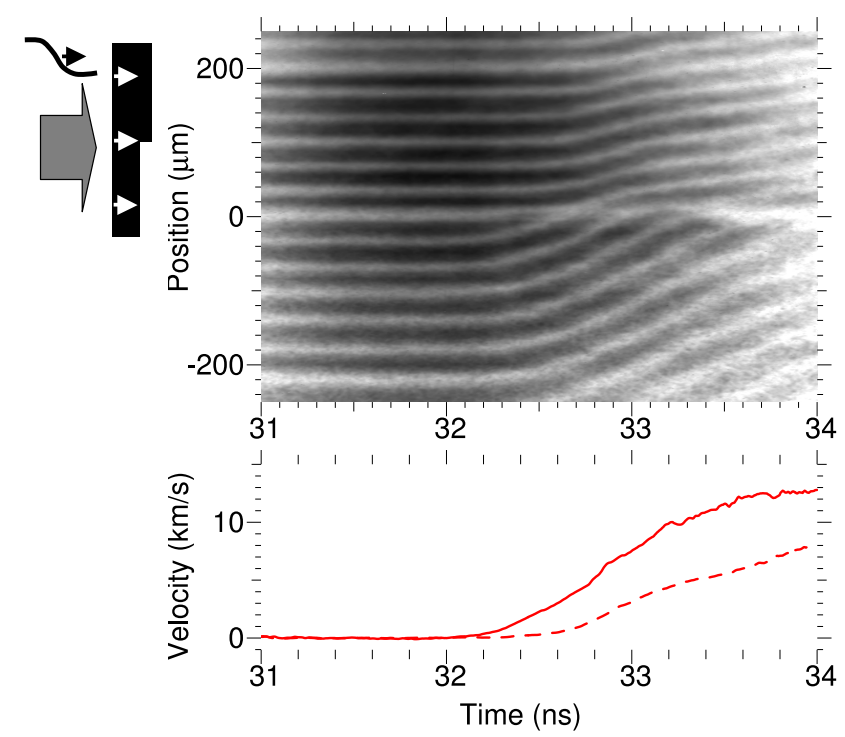

FIG. 9: Free surface motion on the rear surface of a stepped Al plate driven with a ramping (shockless) stress wave. The curves show the velocities extracted from the thin (solid curve) and thick (dashed curve) steps of the foil. 


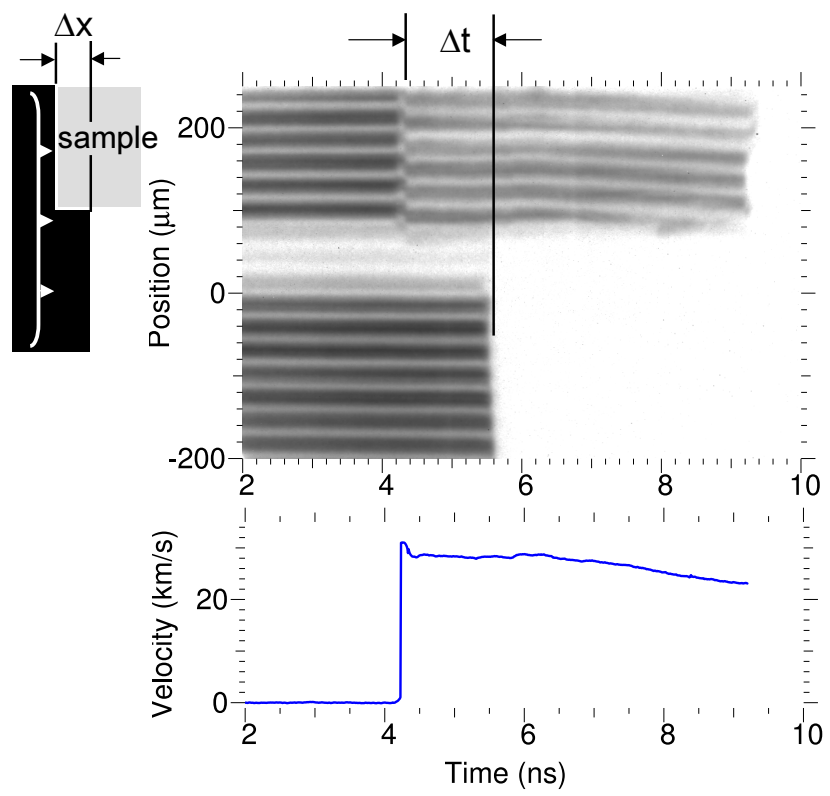

FIG. 10: Impedance matching equation of state experiment, during which a shock is transmitted into the transparent sample through the lower step of a stepped Al baseplate. The lower frame shows the velocity of the shock front in the sample as determined from the fringe phase on the upper part of the recording. There is a brief $(\sim 150 \mathrm{ps})$ velocity transient when the the shock is transmitted through an adhesive layer before entering the sample. 


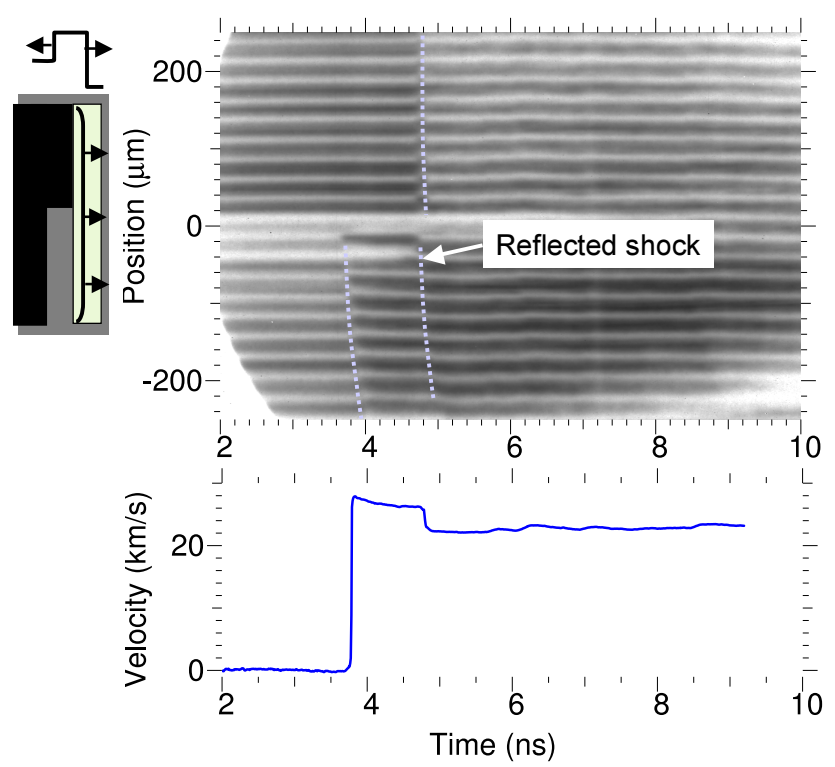

FIG. 11: Anvil reflected shock experiment: a shock incident in an Al plate is transmitted from the lower $\mathrm{Al}$ step into a transparent fluid sample (water) and then into a transparent anvil (fused silica). The anvil is attached to the upper step. The lower frame shows the shock velocities in the fluid sample and in the anvil. 


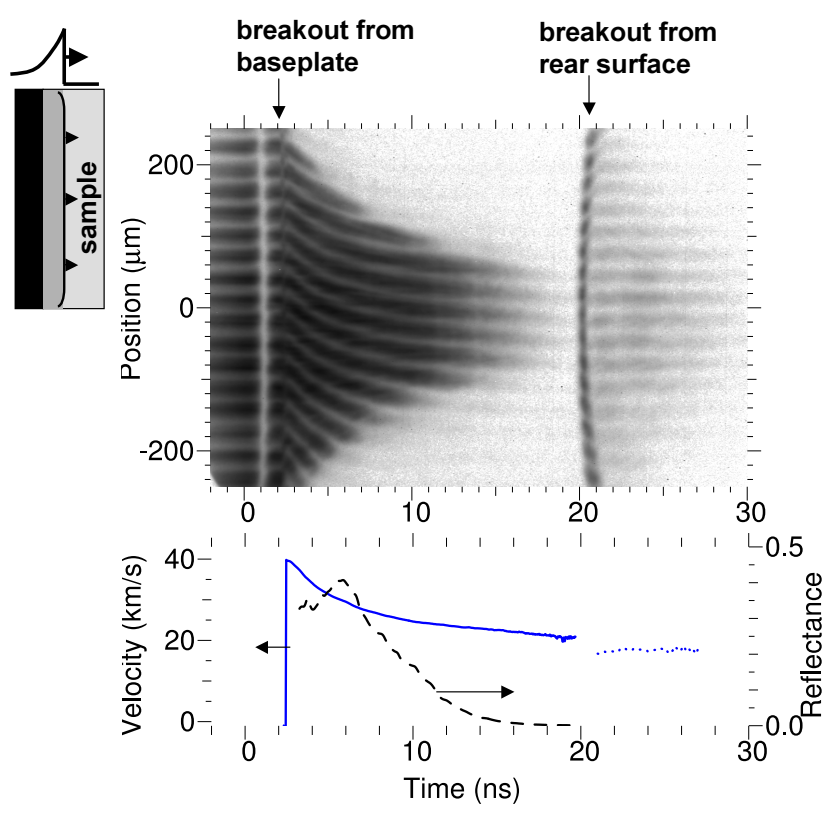

FIG. 12: Attenuating shock in diamond. In the lower frame are shown the velocity of the shock front (solid curve) and the velocity of the free surface of the diamond crystal after release (dotted curve). The reflectance of the shock front (dashed curve) is also shown on the same plot. 


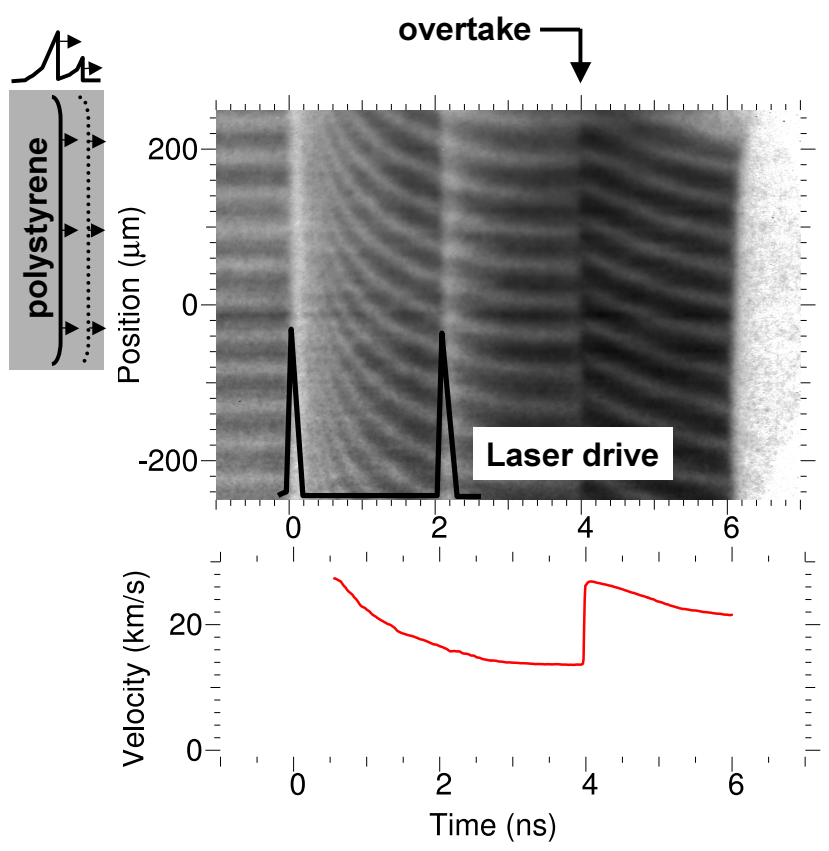

FIG. 13: Multiple attenuating shocks launched in a polystyrene sample by two short duration intense pulses separated by a 2 ns interval. The lower frame (solid curve) shows the velocity of the leading shock front. 